\title{
Side Effects of Chemotherapy in Cancer Patients and Evaluation of Patients Opinion about Starvation Based Differential Chemotherapy
}

\author{
Muhammad Shahbaz Aslam1*, Sidra Naveed"1, Aftab Ahmed², Zaigham Abbas², Iram Gull1, \\ Muhammad Amin Athar ${ }^{1}$ \\ ${ }^{1}$ Institute of Biochemistry \& Biotechnology, University of the Punjab, Lahore, Pakistan \\ ${ }^{2}$ Department of Microbiology and Molecular Genetics, University of the Punjab, Lahore, Pakistan \\ Email: Shahbaz.ibb@pu.edu.pk
}

Received 7 May 2014; revised 3 June 2014; accepted 1 July 2014

Copyright (C) 2014 by authors and Scientific Research Publishing Inc.

This work is licensed under the Creative Commons Attribution International License (CC BY).

http://creativecommons.org/licenses/by/4.0/

(c) (i) Open Access

\section{Abstract}

Side-effects associated with the cancer chemotherapy limit the scope of chemotherapeutic drugs and no data was available about these side effects in Pakistan. Moreover starvation based differential chemotherapy has been proved to greatly reduce the side effects of chemotherapy depending on starvation time. The current study was conducted to survey the common side effects of the chemotherapeutic drugs and the role of starvation to reduce them. The study included total 100 subjects with multiple carcinomas. A comprehensive questionnaire about starvation inquiry, chemotherapy side effects and their basic information was filled by interviewers as told by patients. There were $48 \%$ patients with breast cancer and $11 \%$ with uterine cancer. Out of these patients $30 \%, 28 \%, 9 \%$ and $9 \%$ patients were agreed to starve for $12,24,36$ and 48 hours respectively. The survey regarding the side effects of chemotherapy showed that $43 \%$ patients were suffering from headache, fatigue $90 \%$, weakness $95 \%$, hair loss $76 \%$, nausea $77 \%$, vomiting $75 \%$, diarrhea $31 \%$, abdominal cramps $40 \%$, mouth sores $47 \%$, dry mouth $74 \%$, memory impairment $14 \%$ and numbness $49 \%$. Breast cancer is the most common cancer in Pakistan. Only $18 \%$ of the total patients were agreed to starve for more than one day. Chemotherapy-associated side effects vary greatly and it does not depend upon cancer type. But these side effects depend on multiple factors such as the type and dose of chemotherapeutic drug, patient's health status and stage of cancer.

\section{Keywords}

Starvation, Differential Chemotherapy, Side Effects, Chemotherapeutic Drugs

\footnotetext{
${ }^{*}$ Corresponding author.
}

How to cite this paper: Aslam, M.S., Naveed, S., Ahmed, A., Abbas, Z., Gull, I. and Athar, M.A. (2014) Side Effects of Chemotherapy in Cancer Patients and Evaluation of Patients Opinion about Starvation Based Differential Chemotherapy. Journal of Cancer Therapy, 5, 817-822. http://dx.doi.org/10.4236/jct.2014.58089 


\section{Introduction}

Cancer is one of the most flourishing diseases of all over the world. Cancer incidences and death rates are rapidly increasing world widely and specially in Pakistan [1]. The cancer occurrence could be associated with various environmental, social, cultural, life-style, hormonal and genetic factors [1]. In addition smoking, reduced physical activity and consumption of highly processed and calorie-rich food are the major causes of cancer. Breast, Colorectal, lung and prostate cancer are most frequently spreading diseases world widely as well as in Pakistan [2]. It is reported that Pakistani population have the highest rate of breast cancer among any Asian population (except Jews in Israel) and also have the highest rates of ovarian cancer of all over the world [3].

Although there are many types of cancer treatments available now, but all of them have some associated side effects. Chemotherapy is the most effective and widely used treatment in most types of malignancies [4]. Firstly it was thought that chemotherapy drugs specifically kill the cancer cells only but now it is well known that it also damages to the normal cells resulting the chemotherapy dose dependent side effects such as fatigue, nausea, hair loss vomiting, etc. and even death may also occur in severe cases. The main strategy of chemotherapy drugs based on the phenomenon that these drugs selectively target the tumor cells, largely by the means of genotoxicity partially caused by the production of reactive oxygen species [5], which does not specifically damages the cancer cells but also the normal cells [6]. Total 132 cancer chemotherapy drugs are approved by the US Food and Drug Administration, of which 56 drugs have been reported to cause oxidative stress [7].

There are some chemoprotectants such as glutathione, amifostine, mesna and dexrazoxane have been reported to provide drug-dependent protection to certain tissue types, but these drugs do not show any increase in overall survival, partially due to the reason that they may also protect cancer cells [8]. Therefore, despite of investigating specifically targeted drugs for cancer cells, there should be a fundamentally novel approach which specially kills the cancer cells but not the normal cells. Starvation has been proved to selectively protect the normal cells but not the cancer cells [9]. Preliminary data suggest that starvation dependent differential chemotherapy is not only feasible and safe in cancer patients but also effective in reducing commonly reported chemotherapy sideeffects.

Dietary restriction (DR) or calorie restriction (CR) has been known to be effective against age related diseases (i.e. diabetes, cardiovascular diseases and most importantly cancer) for a long time in various animal models [10] [11]. Recently starvation has been proved to selectively protect the normal cells and mice but not the cancer cells against a variety of chemotherapy drugs [9] [12]. But up till now there is only a limited evidence of implementation of starvation based differential chemotherapy in literature. A case report of 10 patients reflects that starvation based differential chemotherapy is not only safe and feasible but also reduces chemotherapy associated side effects [13].

The current study was executed to investigate the common side effects of chemotherapy and their relation to different socio-economic factors and patient's opinion about starvation. As starvation based differential chemotherapy seems helpful to alleviate chemotherapy related side effects, but it is very important to know the patients' opinion to accept the new interventions. So, this survey also included the opinion of patients to starve for different time lengths in order to reduce the side effects of their chemotherapeutic treatment.

\section{Materials and Methods}

A comprehensive questionnaire was established, first part of which was related to basic information, socioeconomic status, patients' opinion about starvation for different time lengths and their willingness for starvation based differential chemotherapy. Second part of the questionnaire was about the side effects of the chemotherapy which included 12 easily identifiable and most commonly occurring side effects. The intensity or seriousness of side effects was graded into four categories from 0 to 3 as no side effect, mild, moderate and Severe respectively. The study was carried out in the ANMOL hospital Lahore, during April and May 2012. Totally 100 patients with multiple carcinomas were subjected to the study. Questionnaire was filled by interviewers, as told by patients because most of the patients were illiterate and signed by the patients or their relatives.

To check the statistical significance of our data, statistical tests were applied using SPSS v17.0. ANOVA test was applied individually on each dependent and independent variable to check significant differences among variables. 


\section{Results and Discussion}

This survey included total 100 patients; of which 79 were females and 21 were male. In this group of patients, it was observed that breast cancer (61\%) was the most common and uterine cancer (14\%) was the second common cancer in the women. Whereas in men cancer related to GIT (30\%) was most common. Other cancers included lung cancer, blood cancer, bone cancer, ovarian cancer, throat cancer, oral cancer, bladder cancer etc (each of which constitute $1 \%-3 \%)$.

\subsection{Side-Effects of Chemotherapy}

According to this study the most frequently reported side effects were weakness (95\%), fatigue (90\%), nausea (77\%), hair loss (76\%) and vomiting (75\%). Each of these side effects was experienced by more than $70 \%$ of the patients. Other prominent side effects include mouth sores, dry mouth and numbness whereas diarrhea, abdominal cramps and memory impairment were less commonly occurring side effects. Some other side-effects which are not mentioned in the table were also reported by some patients i.e. temperature, constipation, mood swings and weight loss. Graph as shown in Figure 1 indicates the number of patients suffering from each of the 12 side effects surveyed in this study.

The complete description of the chemotherapy side effects is described in the Table 1 . It was observed that

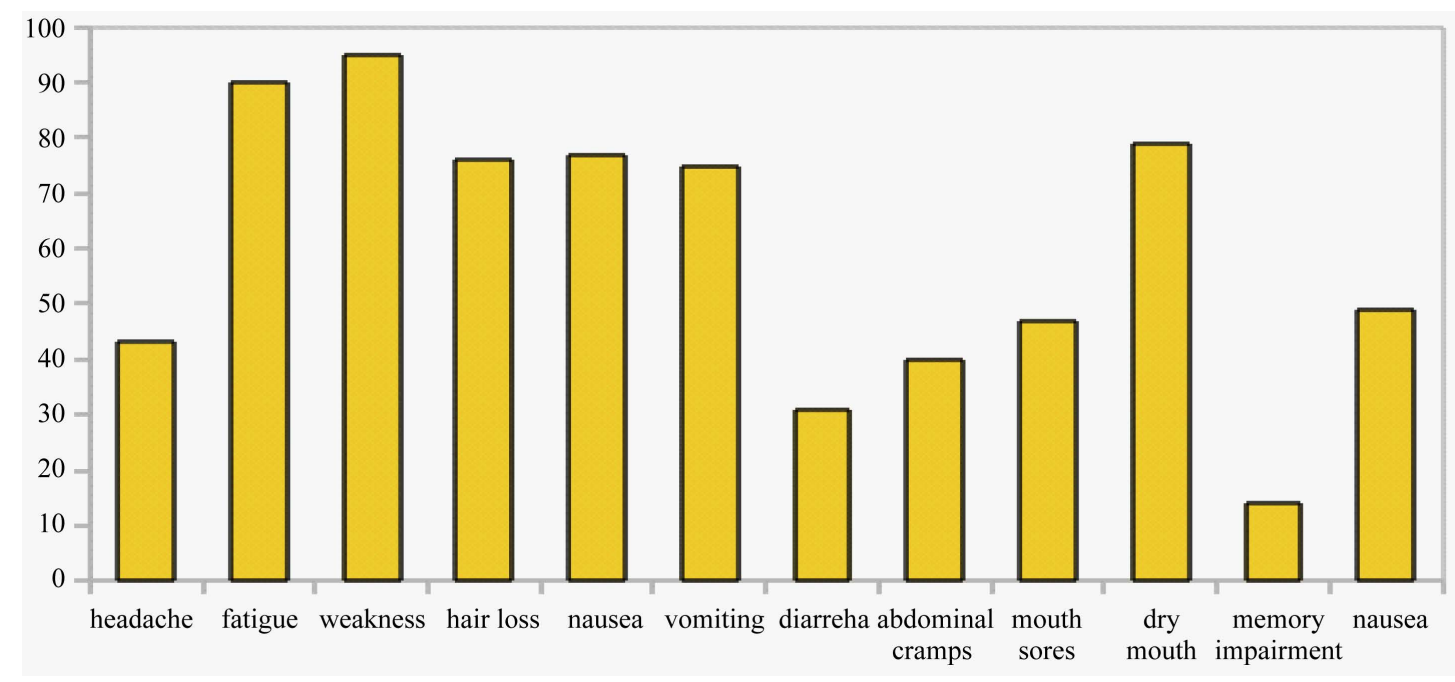

Figure 1. Graph showing the prevalence of different side effects of chemotherapeutic drugs among cancer patients having multiple carcinomas.

Table 1. Most commonly occurring side effects associated with chemotherapy and their level of severity.

\begin{tabular}{|c|c|c|c|c|c|}
\hline Sr. no. & Side effects & No $(\%)$ & Mild (\%) & Moderate (\%) & Severe (\%) \\
\hline 1 & Headache & 57 & 14 & 23 & 6 \\
\hline 2 & Fatigue & 10 & 18 & 37 & 35 \\
\hline 3 & Weakness & 5 & 12 & 34 & 49 \\
\hline 4 & Hair loss & 24 & 8 & 16 & 52 \\
\hline 5 & Nausea & 23 & 15 & 37 & 25 \\
\hline 6 & Vomiting & 25 & 22 & 26 & 27 \\
\hline 7 & Diarrhea & 69 & 8 & 15 & 8 \\
\hline 8 & Abdominal cramps & 60 & 22 & 13 & 5 \\
\hline 9 & Mouth sores & 53 & 14 & 18 & 15 \\
\hline 10 & Dry mouth & 21 & 12 & 43 & 24 \\
\hline 11 & Memory impairment & 86 & 7 & 7 & 0 \\
\hline 12 & Numbness & 51 & 20 & 25 & 4 \\
\hline
\end{tabular}


$25 \%$ - 50\% patients were suffering from severe fatigue, weakness, hair loss, nausea vomiting and dry mouth. While headache, diarrhea, abdominal cramps, memory impairment and and numbness was not much severe.

Statistical tests were applied to check the significance of data and to check whether there is any significant difference in headache, fatigue, weakness, hair loss, Nausea, Vomiting, diarrhea, abdominal cramps, mouth sores, dry mouth, memory impairment and numbness among patients on the basis of gender, cancer type, its duration, other diseases, starvation time, financial status, smoking, number of cigarette number, profession, residence and physical health.

ANOVA test was performed for all the variables on the basis of Gender, cancer type, its duration, other diseases, starvation time, financial status, smoking, number of cigarettes, profession, residence and physical health. Significant difference was seen in male and females for hair loss and Mouth Soar while no difference was seen in other variables on the basis of gender. $66 \%$ men have lost the hairs while $78 \%$ of the women were suffered with hair loss. $28.6 \%$ men were suffering from mouth sores while $58.9 \%$ of the women were affected as shown in Figure 2.

A significant difference in hair loss was also seen among various cancer types. There was a significant difference in headache, fatigue and weakness when variables were checked on the basis of duration of cancer. It is easily understood that as the duration of cancer increases and hence the chemotherapy cycles which leads to the most common side effects specially headache, fatigue and weakness. During chemotherapy treatment where cancer cells are destroyed, normal cells are also affected. Fatigue and weakness are the primary symptoms of any destruction in the body. So as these problems are increased, headache, fatigue and weakness are also increased.

Significant difference was seen in diarrhea condition when various other diseases were compared among cancer patients. Other diseases experienced by the patients include diabetes, hypertension, blood pressure, cardiac problems, stomach problem and lung problem. Notably diarrhea was observed in the patients already suffering from other diseases, specially patients with diabetes, heart, stomach and lung problems. It might be possible that these diseases may contribute to the diarrhea as a side effect of chemotherapy but there is no solid reason or proof in the literature.

But significant difference was found in numbness when patients were compared on the basis of cigarette number used. Significant difference was seen in diarrhea, abdominal cramps and memory impairment when patients were compared on the basis of their profession. The female patients other than housewives with any other profession and some of the males were suffering from all three or two of above diseases collectively. Significant difference was also observed in memory impairment when residence of patients was compared. Among 14 memory impairment cases only 2 were rural while all others were urban. There was no difference in any variable when they were compared on the basis of physical health. Significant difference was also seen in abdominal cramps and mouth sores when patients were divided into three age groups, teenagers, adults and old. Teenagers were most subjected to abdominal cramps and mouth sores.

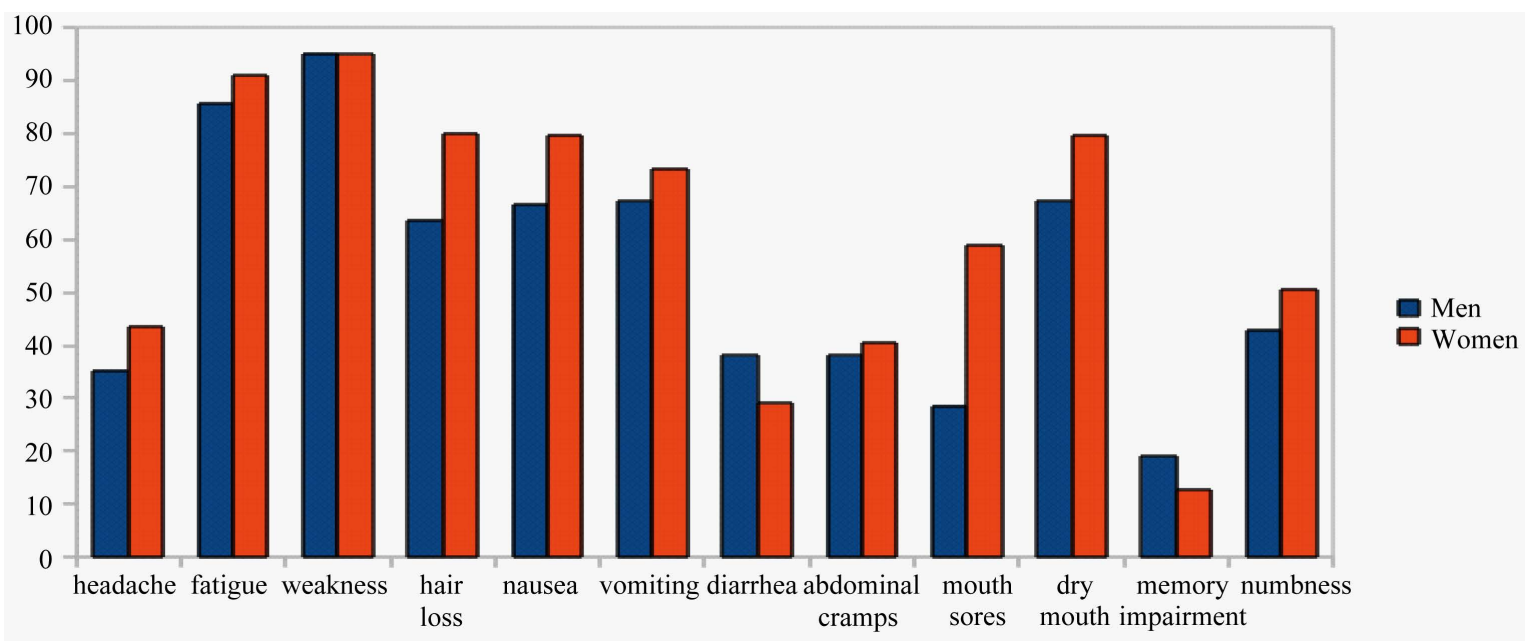

Figure 2. Comparison of the two genders on the basis of different side effects associated with chemotherapeutic drugs. 


\subsection{Starvation and Differential Chemotherapy}

The patients included in this study were briefed about the starvation and its role to alleviate chemotherapy side effects on the basis of current literature. As it is mentioned earlier that more than $80 \%$ of the patients were illiterate so they were totally dependent on the doctors. Most of the patients said that if doctors suggest them that it is reliable and starvation actually reduces the chemotherapy side effects then they have no objection. Then it was asked that for how long the patients may starve? On the whole $76 \%$ of the patients were agreed for starvation, but only 18\% were willing to starve for more than $24 \mathrm{hrs}$ (no $=24 \%, 12 \mathrm{hrs}=30 \%$, $24 \mathrm{hrs}=28 \%, 36 \mathrm{hrs}=9 \%$, $48 \mathrm{hrs}=9 \%$ ) as shown in Figure 3.

When starvation time was compared among patients then significant difference was observed in fatigue of the patients. It was observed that the patients with relatively high level of fatigue completely refused for starvation. $100 \%$ of the patients who denied starving were suffered from a high degree of fatigue.

\subsection{Conclusions and Future Aspects}

Cancer is one of the diseases which are flourishing very rapidly throughout the world due to which mortality rate is also going to increase world widely and particularly in Pakistan [1]. Mostly cancer treatments are based on selective killing of the cancer cells but not normal cells. Chemotherapy is the most widely used treatment for a large number of cancer types. Earlier these chemotherapeutic drugs were considered to be quite targeted and selective for tumor cells, but now it is a well-known that normal cells are also damaged by chemotherapeutic drugs, which leads to various side effects and in some cases even death [6]. These side effects include headache, fatigue, weakness, hair loss, nausea, vomiting, diarrhea, abdominal cramps, mouth sores, dry mouth, memory impairment and Numbness.

In this study we have evaluated the patients with various types of cancers for chemotherapy side-effects. These side effects were subjected to statistical test to determine their relation with different variables, but these results are less reliable due to the absence of common factor in the patients. As this survey included a great variety in cancer types, disease time, age of patient, chemotherapy cycle and the drug being used, so there was a great variation in results. If the patients of a same group are considered then the actual relation of side effects could be derived with other variable like gender, age, other diseases etc. Somehow it is a reality that chemotherapy side-effects are highly disastrous. These side effects limit the effect of chemotherapy. So, there is a need of novel cancer treatment approach, which specifically reduces the tumor with minimum or no side effects.

Starvation has been shown to selectively kill the cancer cells but protect the normal cells and mice, against oxidants and common chemotherapeutic drugs [9] [14] [15]. Early studies suggest that fasting is not only safe and feasible in cancer patients, but also seems to be effective in reducing common chemotherapy associated side effects [13]. Normally increased food intake is prescribed to the cancer patients undergoing chemotherapy to avoid weight loss and other complications. But now it has been proved that excessive food intake with low physical activity, greatly increases the cancer risk [7], as cancer patients have already lower physical activity.

However, short-term starvation as studied in yeast, mice and cell lines, has a great potential to differentially protect normal cells as compared to other interventions. But, further studies and clinical trials should be con-

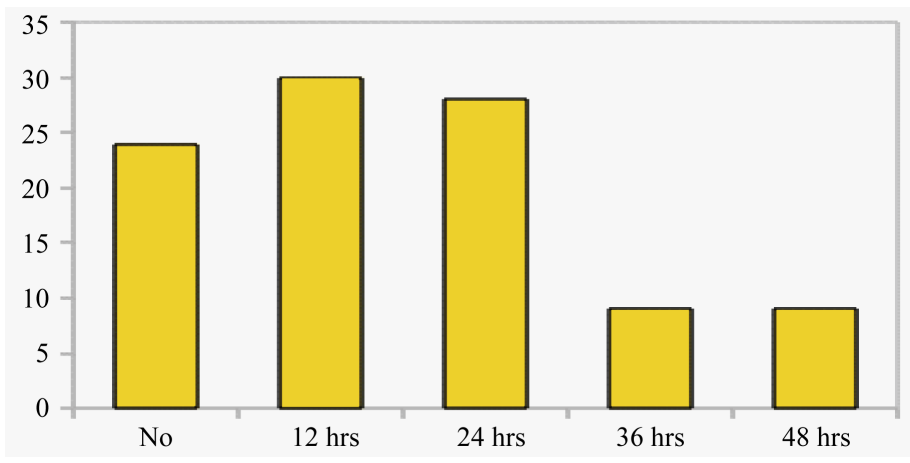

Figure 3. Graph showing no cancer patients willing for starvation for different time lengths $(0,12,24,36$ and 48 hours) in order to reduce the side effects associated with chemotherapy. 
ducted to evaluate the efficacy of differential chemotherapy. In the meanwhile, dietary supplements with calories as low as possible or food deficient of specific macronutrients i.e. glucose or protein, may be helpful in better cancer treatments. Furthermore there is a need of counseling between scientists, doctors and patients as most of our doctors are unknown to the latest research and hence do not accept any new intervention. A great population of Pakistan is illiterate and is unable to understand the mechanism of any intervention or therapy, so are totally dependent on doctors. So there should be a collaborated study in which scientists, doctors and educated patients would participate and work together to study the pros and cons of the starvation based differential chemotherapy.

\section{References}

[1] Javed, S., Ali, M., Sadia, S., Aslam, M.A., Masood, A.I., Shaikh, R.S. and Sayyed, A.H. (2011) Combined Effect of Menopause Age and Genotype on Occurrence of Breast Cancer Risk in Pakistani Population. Maturitas, 69, 377-382. http://dx.doi.org/10.1016/j.maturitas.2011.05.008

[2] Khokher, S., Qureshi, W., Mahmood, S., Saleem, A. and Mahmud, S. (2011) Knowledge, Attitude and Preventive Practices of Women for Breast Cancer in the Educational Institutions of Lahore, Pakistan. Asian Pacific Journal of Cancer Prevention, 12, 2419-2424.

[3] Liede, A., Malik, I.A., Aziz, Z., De Los Rios, P., Kwan, E. and Narod, S.A. (2002) Contribution of BRCA1 and BRCA2 Mutations to Breast and Ovarian Cancer in Pakistan. American Journal of Human Genetics, 71, 595-606. http://dx.doi.org/10.1086/342506

[4] Chabner, B.A. and Roberts Jr., T.G. (2005) Timeline: Chemotherapy and the War on Cancer. Nature Reviews Cancer, 5, 65-72. http://dx.doi.org/10.1038/nrc1529

[5] Conklin, K.A. (2004) Chemotherapy-Associated Oxidative Stress: Impact on Chemotherapeutic Effectiveness. Integrative Cancer Therapies, 3, 294-300. http://dx.doi.org/10.1177/1534735404270335

[6] Lee, C. and Longo, V.D. (2011) Fasting vs Dietary Restriction in Cellular Protection and Cancer Treatment. Oncogene, 30, 3305-3316. http://dx.doi.org/10.1038/onc.2011.91

[7] Chen, Y., Jungsuwadee, P., Vore, M., Butterfield, D.A. and St Clair, D.K. (2007) Collateral Damage in Cancer Chemotherapy: Oxidative Stress in Nontargeted Tissues. Molecular Interventions, 7, 147-156. http://dx.doi.org/10.1124/mi.7.3.6

[8] Links, M. and Lewis, C. (1999) Chemoprotectants: A Review of Their Clinical Pharmacology and Therapeutic Efficacy. Drugs, 57, 293-308. http://dx.doi.org/10.2165/00003495-199957030-00003

[9] Raffaghello, L., Lee, C., Safdie, F.M., Wei, M., Madia, F., Bianchi, G., et al. (2008) Starvation-Dependent Differential Stress Resistance Protects Normal but Not Cancer Cells against High-Dose Chemotherapy. The National Academy of Sciences of the USA, 105, 3305-3316.

[10] Tannenbaum, A. (1945) The Dependence of Tumor Formation on the Composition of the Calorie-Restricted Diet as Well as on the Degree of Restriction. Cancer Research, 5, 616-625.

[11] Hursting, S.D., Perkins, S.N. and Phang, J.M. (1945) Calorie Restriction Delays Spontaneous Tumorigenesis in p53-Knockout Transgenic Mice. Proceedings of the National Academy of Sciences of the USA, 91, 7036-7040. http://dx.doi.org/10.1073/pnas.91.15.7036

[12] Lee, C., Raffaghello, L., Brandhorst, S., Safdie, F.M., Bianchi, G., Montalvo, A.M., et al. (2012) Fasting Cycles Retard Growth of Tumors and Sensitize a Range of Cancer Cell Types to Chemotherapy. Science Translational Medicine, 4, 124-127. http://dx.doi.org/10.1126/scitranslmed.3003293

[13] Safdie, F.M., Dorff, T., Quinn, D., Fontana, L., Wei, M., Lee, C., et al. (2009) Fasting and Cancer Treatment in Humans: A Case Series Report. Aging, 1, 988-1007.

[14] Longo, V.D., Ellerby L.M., Bredesen, D.E., Valentine, J.S. and Gralla, E.B. (1997) Human Bcl-2 Reverses Survival Defects in Yeast Lacking Superoxide Dismutase and Delays Death of Wild-Type Yeast. The Journal of Cell Biology, 137, 1581-1588. http://dx.doi.org/10.1083/jcb.137.7.1581

[15] Longo, V.D. and Fontana, L. (2010) Calorie Restriction and Cancer Prevention: Metabolic and Molecular Mechanisms. Trends in Pharmacological Sciences, 31, 89-98. http://dx.doi.org/10.1016/j.tips.2009.11.004 
Scientific Research Publishing (SCIRP) is one of the largest Open Access journal publishers. It is currently publishing more than 200 open access, online, peer-reviewed journals covering a wide range of academic disciplines. SCIRP serves the worldwide academic communities and contributes to the progress and application of science with its publication.

Other selected journals from SCIRP are listed as below. Submit your manuscript to us via either submit@scirp.org or Online Submission Portal.
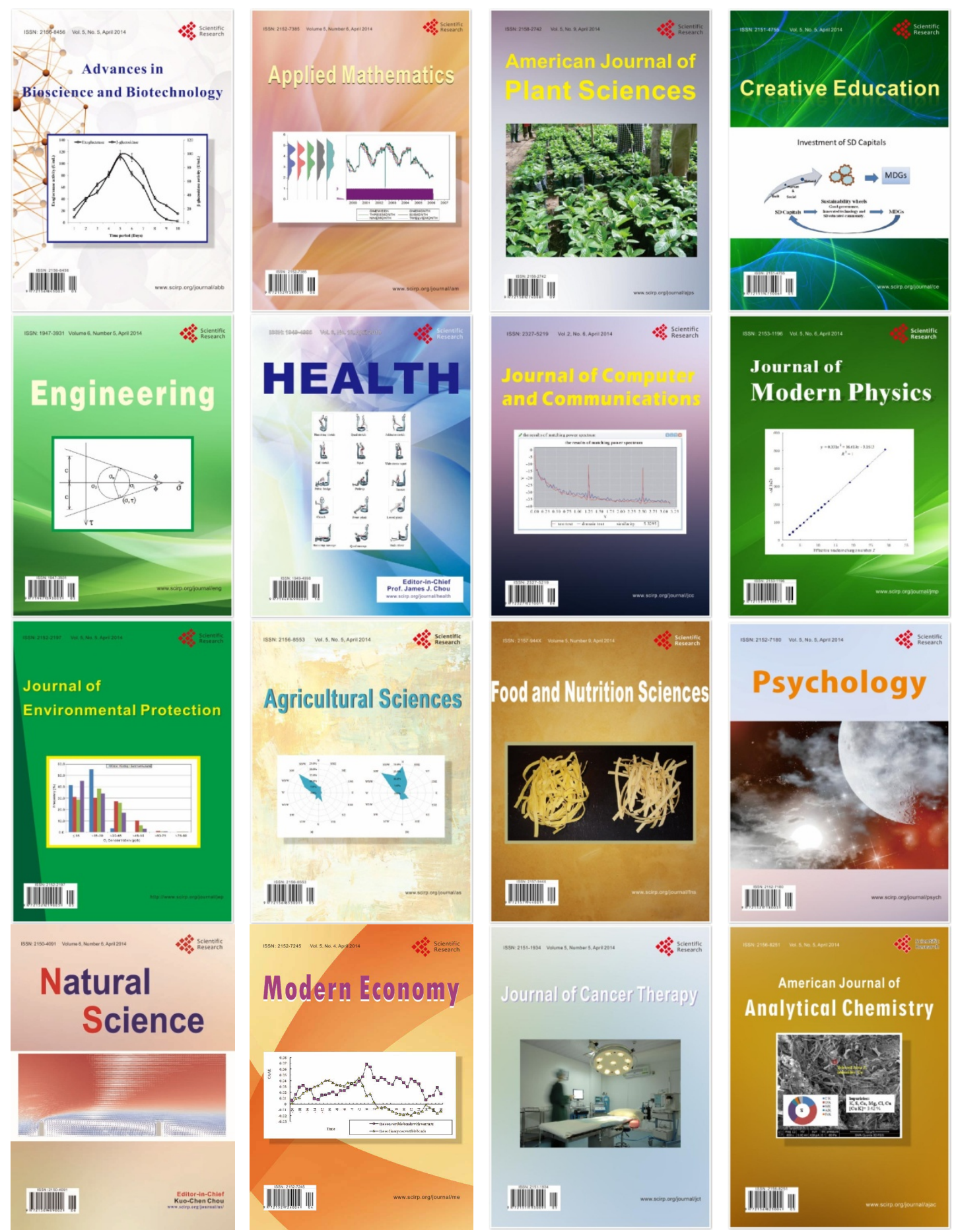